\title{
Agroecossistemas de produção familiar da Amazônia e seus impactos nos atributos do solo ${ }^{1}$
}

\author{
Idelfonso C. de Freitas ${ }^{2}$, Felipe C. V. dos Santos ${ }^{3}$, Ronaldo de O. Custódio Filho ${ }^{3}$, \\ Vladia Correchel ${ }^{4}$ \& Ruy B. da Silva ${ }^{2}$
}

\begin{abstract}
RESUMO
A ausência de restrições climáticas para a produção biológica é a principal característica do bioma Amazônia, o que não se traduz em elevadas produtividades por sucessivos anos de cultivos quando a floresta é substituída por agroecossistemas. O objetivo deste estudo foi avaliar o efeito do manejo do solo nos atributos químicos e físicos do solo pela agricultura familiar, conduzida de forma primitiva. As maneiras de uso avaliadas foram: sistema agroflorestal, pastagem cultivada, roça de toco e mata preservada (usada como referência), todas em Neossolo Quartzarênico. Monólitos de terra foram coletados nas camadas de 0-5, 5-10, 10-20 e 20-40 cm em dois anos agrícolas consecutivos (2010/2011 e 2011/2012) nos meses de julho e janeiro coletando-se sete repetições por sistema. Os atributos do solo avaliados foram: grau de floculação, agregados maior que $2 \mathrm{~mm}$, matéria orgânica, acidez, bases trocáveis, teores de fósforo, saturação por bases, alumínio e capacidade de troca de cátions. Os resultados apontam que 22 anos após a conversão da floresta em agroecossistemas ainda é possível verificar melhores indicadores nos sistemas manejados.
\end{abstract}

Palavras-chave: efeito das cinzas, indicadores de qualidade, propriedades químicas

\section{Agroecosystems of family production in the Amazon and its impacts on soil attributes}

\begin{abstract}
The absence of environmental constraints to organic production is the main characteristic of the Amazon, which does not translate into high yields of crops for several successive years when the forest is replaced by agroecosystems. The objective of this study was to evaluate the effect of soil management on the chemical and physical properties of soil by family agriculture, conducted in primitive way. The evaluated forms of use were: agroforestry system, pasture, slash-and-burn agriculture and preserved forest (used as reference), all under Entisol Quartzipsamment. Soil monoliths were collected at 0-5, 5-10, 10-20 and 20-40 cm during two consecutive years (2010/2011 and 2011/2012) in July and January, collecting seven replicates per system. The evaluated soil attributes were: flocculation, aggregates larger than $2 \mathrm{~mm}$, organic matter, acidity, exchangeable cations, phosphorus, base saturation, aluminum and cation exchange capacity. The results indicate that 22 years after the conversion of forest into agricultural ecosystems it is still possible to see the best indicators in managed systems.
\end{abstract}

Key words: effect of ashes, quality indicators, chemical properties

Parte da Tese de Doutorado do primeiro autor apresentada ao Programa de Pós-Graduação em Agronomia da UFG (Solo e Água)

2 IFTO - Campus Araguatins, Pov. Sta. Tereza, km 05, Zona Rural, S/N, CEP 77950-000, Araguatins, TO. Fone: (63) 3474-4800. E-mail: idelfonsocolares@uol.com.br; ruyborgess@yahoo.com.br

${ }^{3}$ EA/UFG - Programa de Pós-Graduação em Agronomia (Solo e Água). Rod. Goiânia/Nova Veneza, Km 0, Campus Universitário II, CEP 79001-970, Goiânia, GO. Fone: (62) 3521-1542. E-mail: felipecvsantos@hotmail.com; rocf1983@gmail.com; vladiacorrechel@hotmail.com

${ }^{4}$ UFG, Campus II. E-mail: vladiacorrechel@hotmail.com 


\section{INTRODUÇÃO}

Em relação a outros ecossistemas terrestres, o bioma amazônico se caracteriza pela ausência de restrições climáticas para a produção biológica, principalmente no que concerne à temperatura, luminosidade e umidade adequadas ao desenvolvimento de plantas e animais (Gehring, 2006). No entanto, a cobertura pedológica da região é, de modo geral, caracterizada por solos ácidos, com baixa capacidade de troca de cátions e reduzida capacidade de disponibilizar água para as plantas (Ferreira et al., 2006). Nessas condições, a floresta tropical da Amazônia retém a maior parte dos nutrientes do ecossistema na sua biomassa (Moura, 2004) e sua exuberância é fundamentada num processo de reciclagem de nutrientes com ciclo quase fechado, que se contrapõem à lixiviação das bases no perfil do solo pelas precipitações intensas e frequentes (Ferreira et al., 2006).

A conversão dessas florestas em agroecossistemas implica na redução da biomassa do sistema (Chioderoli et al., 2012), o que leva à quebra do equilíbrio no processo de reciclagem de nutrientes (Luizão et al., 2006) e redução dos teores de matéria orgânica do solo. Isto tem consequência na nutrição das plantas por reduzir os sítios de troca do complexo coloidal do solo (Silva et al., 2011) e, assim, expor as bases trocáveis aos efeitos da lixiviação favorecida pela alta permeabilidade do solo associada a precipitações torrenciais, característica da região (Lima et al., 2011). Essas consequências são mais evidentes quando o preparo do solo é fundamentado na aração, na gradagem (Chioderoli et al., 2012; Matoso et al., 2012) e na agricultura de corte e queima da vegetação natural (Iwata et al., 2012).

Neste cenário, o grande desafio na Amazônia tem sido desenvolver agroecossistemas capazes de conciliar, de forma harmoniosa, interesses de conservação ambiental com sustentabilidade econômica em substituição à agricultura de corte e queima da vegetação natural comumente praticada (Menezes et al., 2008). É provável que, por representar uma forma de ocupação do solo pouco onerosa para o produtor e pelos efeitos da cinza proveniente da queima da vegetação espontânea no $\mathrm{pH}$ e na disponibilidade de bases necessárias à nutrição das culturas, um enorme contingente de produtores, sobretudo os de produção familiar, haja optado por esse tipo de preparo do solo (Moura, 2004).

A maioria dos trabalhos relacionados à agricultura itinerante (corte e queima) menciona que os nutrientes disponibilizados pela queima das florestas nativas na Amazônia, usualmente suportam de dois a três anos com cultivos anuais. Passado este período, a produtividade das culturas decai drasticamente obrigando os produtores a abandonarem as áreas em pousio florestal para formação de capoeiras, invariavelmente mais pobres em diversidade biológica e biomassa quando comparada com as florestas originais (Dubois et al., 1996).

Pesquisas recentes têm apontado os sistemas agroflorestais como alternativa promissora visando à agricultura familiar no trópico úmido (Maia et al., 2006; Lima et al., 2011; Iwata et al., 2012), em especial para a região amazônica (Menezes et al., 2008). Associada ao cultivo simultâneo com espécies florestais, a diversidade de espécies agrícolas parece ser a chave do sucesso na manutenção da fertilidade do solo (Silva et al., 2011) possivelmente por se assemelhar à estrutura florística das florestas nativas. Segundo esses autores, somente manter a fertilidade natural do solo não é suficiente para garantir a sustentabilidade do sistema uma vez que, nessas condições, existem exportações de nutrientes por meio da retirada dos frutos e grãos para consumo e comercialização, exaurindo as reservas naturais de nutrientes do solo.

Neste sentido, pesquisas envolvendo a dinâmica dos nutrientes em sistemas agroflorestais com longo histórico de uso são necessárias para esclarecer a suposta sustentabilidade que lhes é atribuída. Assim, o propósito deste trabalho foi avaliar as propriedades químicas de um Neossolo Quartzarênico Órtico com manejo da agricultura familiar na região amazônica, cultivado em regime de sistema agroflorestal, pastagem cultivada e roça de toco em comparação a uma floresta preservada.

\section{Material e Métodos}

A área experimental está localizada no lote 52 do assentamento Tobasa, em Esperantina, TO, nas coordenadas $5^{\circ} 22^{\prime} 02^{\prime \prime}$ de latitude sul, $48^{\circ} 35^{\prime} 57^{\prime \prime}$ de longitude a oeste de Greenwich e $90 \mathrm{~m}$ de altitude. A precipitação média local é de $1.500 \mathrm{~mm} \mathrm{ano}^{-1} \mathrm{e}$ a temperatura média de $28,5^{\circ} \mathrm{C}$. O clima, classificado como Aw (Köppen), apresenta seis meses de período chuvoso (dezembro a maio) e seis meses de período seco (junho a novembro).

O solo da área foi classificado como Neossolo Quartzarênico Órtico $\left(\mathrm{RQ}_{0}\right)$, conforme EMBRAPA (2006) e se encontra sob quatro usos distintos: i) SAF: sistema agroflorestal implantado em 1989 em volta da sede, que evoluiu de um quintal para uma agrofloresta com mais de sessenta espécies cultivadas em consórcio, sendo o cupuaçu (Theobroma grandiflorum) a principal cultura econômica do sistema seguido pela do bacuri (Platonia insignis) que ocupam uma área de três hectares; ii) PA: pastagem cultivada implantada em 1989 com capim marmelada (Brachiaria plantaginea), localizada ao lado do sistema agroflorestal com área aproximada de 30 hectares; iii) RT: roça de toco de 2,50 hectares, caracterizada pela retirada da floresta nativa que teve sua fitomassa seca queimada em 2009, sendo utilizada para cultivo com arroz (Oryza sativa), milho (Zea mays), feijão trepa-pau (Vigna unguiculata) e mandioca (Manihot esculenta) até maio de 2010 e mantida em pousio após a colheita dessa área até o momento e iv) MA: mata nativa localizada ao lado da roça de toco usada, neste estudo, como área de referência. Todos os agroecossistemas foram iniciados com corte e queima da vegetação natural. Não havia registro do uso de mecanização nem de adubos solúveis para nenhum dos sistemas estudados.

Em cada sistema de uso do solo foi delimitada uma transeção na qual foram coletadas amostras de solo em sete pontos distantes $10 \mathrm{~m}$ entre si. A amostragem foi feita durante dois anos e em duas épocas: período seco (julho/2010 e 2011) e período chuvoso (janeiro/2011 e 2012), resultando em quatro amostragens. No entorno de cada ponto de amostragem foi aberta uma minitrincheira em cujas paredes foram coletados e acondicionados em filme plástico, monólitos de terra de $5 \mathrm{x}$ 
20 x $20 \mathrm{~cm}$ nas camadas de $00-05,05-10,10-20$ e $20-40 \mathrm{~cm}$. No laboratório os monólitos foram secados ao ar até atingir peso constante; depois de secos, foram destorroados e passados em uma sequência de peneiras com abertura de 4 e $2 \mathrm{~mm}$; os agregados retidos na peneira de $2 \mathrm{~mm}$ foram selecionados para análise de estabilidade e o material retido na peneira sem abertura foi para análise granulométrica e química da terra.

Todas as análises foram realizadas de acordo com a metodologia descrita em EMBRAPA (1997). A textura foi determinada pelo método do densímetro; o grau de floculação foi obtido pela relação entre argila naturalmente dispersa e a argila total, obtida após dispersão; a porcentagem de agregados por tamanho foi determinada por peneiramento lento via úmida; MOS - oxidação via úmida com solução de dicromato de potássio em meio ácido utilizando-se, como fonte externa de calor, o ácido sulfúrico e titulado com sal de Mohr; $\mathrm{pH}$ - extraído em $\mathrm{CaCl}_{2} ; \mathrm{H}+\mathrm{Al}$ - acetato de cálcio; $\mathrm{P}$ e $\mathrm{K}$ - Mehlich I e determinados por colorimetria e fotometria de chama, respectivamente e; $\mathrm{Al}^{3+}, \mathrm{Ca}$ e $\mathrm{Mg}$ - espectrofotometria de absorção atômica extraídos com $\mathrm{KCl}$.

Os dados foram submetidos à análise de variância segundo o delineamento inteiramente casualizado e as médias comparadas pelo teste de Scott-Knott $(\mathrm{p}<0,05)$. Para viabilizar a comparação entre sistemas frente ao conjunto de variáveis envolvidas, foi feita análise multivariada pelo método de agrupamento de Ward, com auxilio do aplicativo computacional PAST versão 2,08b (Hammer et al., 2001).

\section{Resultados E Discussão}

Na Tabela 1 se observa que os sistemas de uso do solo estudados estão sobre a mesma classe textural, em todas as camadas. Assim, as diferenças nos atributos físicos e químicos do solo devem ser atribuídas à atividade antrópica efetuada ao

Tabela 1. Distribuição das frações granulométricas argila (ARG), silte (SIL) e areia total (AT), classe textural, argila dispersa em água (AD) e grau de floculação (GF) nas camadas de um Neossolo Quartzarênico Órtico sob diferentes usos da agricultura familiar

\begin{tabular}{|c|c|c|c|c|c|c|c|}
\hline \multirow{2}{*}{$\begin{array}{l}\text { Camada } \\
\text { (cm) }\end{array}$} & \multirow{2}{*}{ Uso ${ }^{1}$} & ARG & SIL & AT & \multirow{2}{*}{$\begin{array}{c}\text { Classe } \\
\text { textural }^{2}\end{array}$} & \multirow{2}{*}{$\underset{\left(g^{\prime} g^{-1}\right)}{A D}$} & \multirow{2}{*}{$\begin{array}{l}\text { GF } \\
(\%)\end{array}$} \\
\hline & & \multicolumn{3}{|c|}{$\left(\mathrm{g} \mathrm{kg}^{-1}\right)$} & & & \\
\hline \multirow{4}{*}{$0-5$} & MA & 113 & 44 & 843 & A.F & 51,43 & 54,08 B \\
\hline & SAF & 111 & 46 & 843 & A.F & 38,57 & $65,30 \mathrm{~A}$ \\
\hline & PA & 117 & 43 & 840 & A.F & 61,43 & $47,41 \mathrm{~B}$ \\
\hline & RT & 123 & 60 & 817 & A.F & 58,57 & $52,45 \mathrm{~B}$ \\
\hline \multirow{4}{*}{$5-10$} & MA & 133 & 42 & 825 & A.F & 47,14 & $63,97 \mathrm{~A}$ \\
\hline & SAF & 110 & 37 & 853 & A.F & 37,14 & $66,42 \mathrm{~A}$ \\
\hline & PA & 113 & 47 & 840 & A.F & 61,43 & $45,32 \mathrm{~B}$ \\
\hline & RT & 126 & 54 & 820 & A.F & 60,00 & $52,04 \mathrm{~B}$ \\
\hline \multirow{4}{*}{$10-20$} & MA & 133 & 43 & 824 & A.F & 58,43 & $55,98 \mathrm{~A}$ \\
\hline & SAF & 111 & 42 & 847 & A.F & 41,43 & $63,33 \mathrm{~A}$ \\
\hline & PA & 120 & 39 & 841 & A.F & 60,00 & $49,80 \mathrm{~A}$ \\
\hline & $\mathrm{RT}$ & 127 & 53 & 820 & A.F & 61,43 & $50,98 \mathrm{~A}$ \\
\hline \multirow{4}{*}{$20-40$} & MA & 137 & 44 & 819 & A.F & 74,28 & $45,84 \mathrm{~B}$ \\
\hline & SAF & 116 & 48 & 836 & A.F & 51,43 & $55,43 \mathrm{~A}$ \\
\hline & PA & 123 & 34 & 843 & A.F & 60,00 & $50,99 \mathrm{~A}$ \\
\hline & RT & 141 & 42 & 817 & A.F & 65,71 & $53,53 \mathrm{~A}$ \\
\hline
\end{tabular}

'MA - Mata; SAF - Sistema agroflorestal; PA - Pasto e RT - Roça de toco. ${ }^{2}$ A.F - Areia franca. Médias seguidas de mesma letra na coluna não diferem entre si para a mesma camada, de acordo com os resultados do teste de Scott-Knott $(p<0,05)$. $N=7$ longo dos anos. Com relação à argila dispersa em água (AD), já é conhecida sua relação com o grau de floculação (GF), em que um valor menor de AD implica em maior GF (Silva et al., 2011), tal como se observa no sistema agroflorestal (SAF) na camada 00-05 cm, assim como na mata (MA) e SAF de 05-10 $\mathrm{cm}$. Esses resultados devem estar associados aos maiores teores de matéria orgânica do solo (MOS) aportada sobretudo pelos componentes arbóreos desses sistemas, em especial na camada superficial do solo (Iwata et al., 2012).

O fracionamento da fração areia é imprescindível nos estudos pedogeomorfológicos (Burak \& Passos, 2011) pela estreita relação com as propriedades físicas do solo. Neste sentido, elevado teor de areia grossa na granulometria favorece a drenagem do excesso de água enquanto alto teor de areia muito fina pode levar ao selamento pelo avanço deste material nos macroporos diminuindo a capacidade de drenagem do solo e, em consequência, expondo-os aos efeitos erosivos das chuvas (Cey et al., 2009; Pedron et al., 2011). No caso deste estudo a maior concentração da areia foi observada na categoria média seguida da fina, o que ameniza as limitações de ordem física do Neossolo Quartzarênico Órtico (RQo) (Tabela 2).

Tabela 2. Distribuição da frequência da fração areia livre de silte e argila (para uma base de $100 \%$ ) nas camadas de um Neossolo Quartzarênico Órtico sob diferentes usos da agricultura familiar

\begin{tabular}{ccrrccc}
\hline $\begin{array}{c}\text { Camada } \\
\text { (cm) }\end{array}$ & Uso $^{1}$ & $\mathbf{M G}^{2}$ & $\mathbf{G}$ & $\mathbf{M}$ & $\mathbf{F}$ & $\mathbf{M F}$ \\
\cline { 3 - 7 } & MA & 3 & 117 & 480 & 360 & 40 \\
$0-5$ & SAF & 2 & 54 & 480 & 408 & 56 \\
& PA & 2 & 49 & 449 & 414 & 86 \\
& RT & 2 & 50 & 552 & 371 & 25 \\
\hline \multirow{5}{*}{$5-10$} & MA & 2 & 101 & 483 & 369 & 45 \\
& SAF & 2 & 37 & 505 & 397 & 59 \\
& PA & 2 & 55 & 439 & 421 & 83 \\
& RT & 3 & 46 & 549 & 376 & 26 \\
\hline \multirow{5}{*}{$10-20$} & MA & 6 & 115 & 470 & 366 & 43 \\
& SAF & 2 & 36 & 478 & 421 & 63 \\
& PA & 2 & 48 & 439 & 427 & 84 \\
& RT & 3 & 43 & 572 & 357 & 25 \\
\hline \multirow{2}{*}{$20-40$} & MA & 8 & 110 & 483 & 357 & 42 \\
& SAF & 2 & 37 & 472 & 422 & 67 \\
& PA & 1 & 56 & 426 & 434 & 83 \\
& RT & 2 & 32 & 559 & 379 & 28 \\
\hline
\end{tabular}

'MA - Mata; SAF - Sistema agroflorestal; PA - Pasto; RT - Roça de toco. ${ }^{2} \mathrm{MG}$ - muito grossa; G - Grossa; M - Média; F - Fina; MF - Muito fina

Independente do período de amostragem, a MOS decresce no perfil do solo em todas as formas de uso estudadas, o que é atribuído ao não revolvimento do solo (Iwata et al., 2012). Os maiores valores foram observados no sistema SAF no período seco nas camadas de 00-05 e 05-10 cm (Tabela 3) em razão, possivelmente, do estágio de sucessão ecológica em que se encontrava o sistema (capoeira grossa), que possibilita incrementos no acúmulo de biomassa e renovação de suas estruturas mais frequentes em relação a MA (Caldeira et al., 2007). É importante ressaltar que, neste trabalho, as diferenças entre formas de uso do solo nos teores de MOS foram direcionadas em favor dos sistemas mais diversificados no período seco (SAF e MA), o que decorre do aporte mais constante de serrapilheira ao piso do solo (Sanches et al., 2009). 
Tabela 3. Atributos químicos (AQ), matéria orgânica do solo $(\mathrm{MOS})$, acidez ativa $(\mathrm{pH})$, potencial $(\mathrm{H}+\mathrm{Al})$, trocável $\left(\mathrm{Al}^{3+}\right)$, saturação por $\mathrm{Al}(\mathrm{m})$ e fósforo disponível $(\mathrm{P})$ nas camadas $(\mathrm{Z})$ e períodos de amostragem de um Neossolo Quartzarênico Órtico sob diferentes usos da agricultura familiar

\begin{tabular}{|c|c|c|c|c|c|c|c|c|c|c|c|c|c|c|c|c|c|}
\hline \multirow{2}{*}{$A Q$} & \multirow{2}{*}{$\begin{array}{c}Z \\
(\mathrm{~cm})\end{array}$} & \multicolumn{2}{|c|}{$\mathrm{MA}^{1}$} & \multicolumn{2}{|c|}{ SAF } & \multicolumn{2}{|l|}{ PA } & \multirow{2}{*}{\multicolumn{2}{|c|}{ RT }} & \multicolumn{2}{|c|}{ MA } & \multicolumn{2}{|c|}{ SAF } & \multicolumn{2}{|l|}{ PA } & \multicolumn{2}{|l|}{ RT } \\
\hline & & \multicolumn{6}{|c|}{ Período seco } & & & \multicolumn{8}{|c|}{ Período chuvoso } \\
\hline \multirow{3}{*}{$\begin{array}{c}\text { MOS } \\
\left(g_{\mathrm{kg}}^{-1}\right)\end{array}$} & $5-10$ & 22,60 & $\mathrm{Bb}$ & 28,10 & $A b$ & 17,60 & $\mathrm{Ca}$ & 14,30 & $\mathrm{Ca}$ & 14,90 & $A b$ & 15,60 & $A b$ & 18,10 & $\mathrm{Aa}$ & 14,90 & $A b$ \\
\hline & $20-40$ & 11,10 & Ac & 12,10 & Ac & 7,70 & $\mathrm{BC}$ & 7,60 & $\mathrm{Bb}$ & 6,80 & $\mathrm{Ad}$ & 7,80 & Ac & 7,70 & Ac & 7,10 & $\mathrm{Ac}$ \\
\hline & $0-40$ & 21,40 & $A$ & 25,10 & A & 14,70 & $\mathrm{~B}$ & 12,60 & $\mathrm{~B}$ & 13,50 & $A$ & 15,60 & $A$ & 14,60 & A & 13,80 & $\mathrm{~A}$ \\
\hline \multirow{3}{*}{$\begin{array}{c}\mathrm{pH} \\
\left(\mathrm{CaCl}_{2}\right)\end{array}$} & $0-5$ & 4,04 & $\mathrm{Ba}$ & 3,91 & $\mathrm{BC}$ & 4,31 & $\mathrm{Aa}$ & 4,42 & $\mathrm{Aa}$ & 4,01 & $\mathrm{BC}$ & 4,06 & $\mathrm{Bd}$ & 4,54 & $\mathrm{Aa}$ & 4,78 & $\mathrm{Aa}$ \\
\hline & $20-40$ & 4,24 & $\mathrm{Ba}$ & 4,33 & $\mathrm{Aa}$ & 4,39 & $\mathrm{Aa}$ & 4,23 & $\mathrm{Ba}$ & 4,37 & $\mathrm{Ba}$ & 4,51 & $\mathrm{Aa}$ & 4,45 & $\mathrm{Aa}$ & 4,29 & $\mathrm{Cb}$ \\
\hline & $0-40$ & 4,13 & $\mathrm{~B}$ & 4,16 & B & 4,34 & A & 4,22 & $B$ & 4,16 & $B$ & 4,31 & A & 4,45 & A & 4,37 & A \\
\hline \multirow{4}{*}{$\begin{array}{c}\mathrm{H}+\mathrm{Al} \\
\left(\mathrm{cmol}_{\mathrm{c}} \mathrm{dm}^{-3}\right)\end{array}$} & $0-5$ & 7,69 & $\mathrm{Aa}$ & 8,26 & $\mathrm{Aa}$ & 7,05 & $\mathrm{Aa}$ & 6,21 & $\mathrm{Aa}$ & 4,04 & $\mathrm{Aa}$ & 3,56 & $\mathrm{Aa}$ & 3,44 & $A b$ & 3,15 & $\mathrm{Aa}$ \\
\hline & 5-10 & 7,00 & $\mathrm{Aa}$ & 6,81 & $A b$ & 6,86 & $\mathrm{Aa}$ & 6,54 & $\mathrm{Aa}$ & 3,49 & $A b$ & 3,04 & $\mathrm{Aa}$ & 3,76 & $\mathrm{Aa}$ & 3,89 & $\mathrm{Aa}$ \\
\hline & $10-20$ & 6,29 & $\mathrm{Aa}$ & 5,03 & $\mathrm{Bc}$ & 5,67 & $\mathrm{Bb}$ & 6,31 & $\mathrm{Aa}$ & 3,14 & Ac & 3,50 & $\mathrm{Aa}$ & 3,20 & $A b$ & 3,86 & $\mathrm{Aa}$ \\
\hline & $20-40$ & 4,79 & $A b$ & 4,19 & $A C$ & 4,28 & $A C$ & 5,03 & $\mathrm{Aa}$ & 2,52 & $\mathrm{Cd}$ & 2,34 & $\mathrm{Ca}$ & 2,73 & $\mathrm{Bc}$ & 3,07 & $\mathrm{Aa}$ \\
\hline \multirow{2}{*}{$\begin{array}{c}\mathrm{Al}^{3+} \\
\left(\mathrm{cmol}_{\mathrm{c}} \mathrm{dm}^{-3}\right)\end{array}$} & $20-40$ & 0,77 & $\mathrm{Aa}$ & 0,48 & $\mathrm{Bb}$ & 0,51 & $\mathrm{Bb}$ & 0,69 & $\mathrm{Aa}$ & 0,75 & Ac & 0,51 & $A b$ & 0,73 & $\mathrm{Aa}$ & 0,73 & $\mathrm{Aa}$ \\
\hline & $0-40$ & 0,79 & A & 0,61 & B & 0,63 & $\mathrm{~B}$ & 0,65 & $B$ & 1,19 & A & 0,68 & B & 0,61 & $B$ & 0,63 & B \\
\hline \multirow{5}{*}{$\begin{array}{l}\mathrm{m} \\
(\%)\end{array}$} & $0-5$ & 53,93 & $A b$ & 62,07 & $\mathrm{Aa}$ & 50,98 & $\mathrm{Aa}$ & 37,86 & $\mathrm{Bb}$ & 64,61 & $\mathrm{Aa}$ & 64,12 & $\mathrm{Aa}$ & 33,20 & $\mathrm{Bb}$ & 22,83 & $\mathrm{Bb}$ \\
\hline & 5-10 & 63,35 & $\mathrm{Aa}$ & 58,92 & $\mathrm{Aa}$ & 57,68 & $\mathrm{Aa}$ & 59,07 & $\mathrm{Aa}$ & 70,49 & $\mathrm{Aa}$ & 56,64 & $\mathrm{Ba}$ & 49,25 & $\mathrm{Ba}$ & 57,67 & $\mathrm{Ba}$ \\
\hline & $10-20$ & 66,02 & $\mathrm{Aa}$ & 57,89 & $\mathrm{Ba}$ & 57,43 & $\mathrm{Ba}$ & 62,45 & $\mathrm{Aa}$ & 67,92 & $\mathrm{Aa}$ & 57,68 & $\mathrm{Ba}$ & 59,36 & $\mathrm{Ba}$ & 60,93 & $\mathrm{Ba}$ \\
\hline & $20-40$ & 64,14 & $\mathrm{Aa}$ & 50,75 & $\mathrm{Bb}$ & 54,90 & $\mathrm{Ba}$ & 60,06 & $\mathrm{Aa}$ & 63,49 & $\mathrm{Aa}$ & 52,84 & $\mathrm{Ba}$ & 56,33 & $\mathrm{Ba}$ & 64,28 & $\mathrm{Aa}$ \\
\hline & $0-40$ & 61,86 & $A$ & 57,41 & B & 55,25 & B & 54,86 & B & 66,63 & $A$ & 57,86 & $B$ & 49,54 & B & 51,43 & B \\
\hline \multirow{5}{*}{$\begin{array}{c}\mathrm{P} \\
\left(\mathrm{mg} \mathrm{dm}^{-3}\right)\end{array}$} & $0-5$ & 3,86 & $\mathrm{Ba}$ & 4,42 & $\mathrm{Ba}$ & 1,62 & $\mathrm{Ba}$ & 9,48 & $\mathrm{Aa}$ & 4,32 & $\mathrm{Ba}$ & 2,96 & $\mathrm{Ba}$ & 2,51 & $\mathrm{Ba}$ & 16,96 & $\mathrm{Aa}$ \\
\hline & 5-10 & 2,46 & $A b$ & 2,96 & $\mathrm{Aa}$ & 0,83 & $\mathrm{Bb}$ & 3,18 & $A b$ & 2,73 & $\mathrm{Bb}$ & 1,71 & $\mathrm{Bb}$ & 2,23 & $\mathrm{Ba}$ & 5,66 & $A b$ \\
\hline & $10-20$ & 1,59 & $A b$ & 1,84 & $\mathrm{Aa}$ & 0,71 & $\mathrm{Bb}$ & 1,93 & $A b$ & 2,09 & $\mathrm{Bb}$ & 1,25 & $\mathrm{Bb}$ & 2,05 & $\mathrm{Ba}$ & 2,92 & $A b$ \\
\hline & $20-40$ & 1,25 & $A b$ & 2,36 & $\mathrm{Aa}$ & 0,49 & $A b$ & 1,32 & $A b$ & 1,64 & $A b$ & 1,23 & $A b$ & 1,34 & $A b$ & 1,57 & $A b$ \\
\hline & $0-40$ & 2,29 & A & 2,90 & A & 0,92 & B & 3,98 & A & 2,69 & B & 1,79 & B & 2,03 & B & 6,78 & A \\
\hline
\end{tabular}

'MA - Mata; SAF- Sistema agroflorestal; PA - Pasto; RT - Roça de toco. Médias seguidas de mesma letra maiúscula nas linhas não diferem entre sistema de uso para o mesmo período de amostragem e médias seguidas por letras minúsculas iguais nas colunas não diferem entre camadas, de acordo com os resultados do teste de Scott-Knott $(p<0,05)$. N $=7$, dois anos de observação

No período chuvoso observa-se redução nos teores de MOS para todos os sistemas avaliados em relação ao período seco, que é justificado pela maior oferta de umidade. Nas referidas condições, a mineralização dos resíduos é favorecida pela população de decompositores, conforme evidenciado por Ferreira et al. (2006), Lima et al. (2011) e Iwata et al. (2012). No período seco roça de toco (RT) e pasto (PA) apresentaram valores de MOS semelhantes entre si e inferiores aos demais sistemas ao longo de todo o perfil do solo, decorrente do consumo da biomassa pelo fogo (em RT) e pelos animais pastoreados (em PA).

Os valores mais elevados de $\mathrm{pH}$ em PA e RT se devem, sem dúvida, ao aporte de nutrientes provenientes da queima da floresta nativa por ocasião da abertura do terreno para uso agrícola (Menezes et al., 2008). Constata-se que, no PA, para os dois períodos de amostragem o $\mathrm{pH}$ é mais elevado que a área de referência em todas as camadas, o que é atribuído ao tempo decorrido entre a queima e as amostragens, o que permitiu a lixiviação das bases no perfil do solo. Na RT, pelo curto período entre o uso do fogo e as amostragens, o efeito das cinzas no $\mathrm{pH}$ só é evidenciado nos primeiros $5 \mathrm{~cm}$ no período seco e até 10 $\mathrm{cm}$ no período chuvoso.

Os sistemas de uso com maior biomassa vegetal viva (MA e SAF) apresentaram $\mathrm{pH}$ mais ácido que as demais formas de uso, provavelmente por conter grande parte das bases trocáveis integrada à sua estrutura (Menezes et al., 2008) Ressalta-se, então, que o maior teor de matéria orgânica aportada ao solo nesses sistemas não foi capaz de diminuir a acidez do solo, talvez pela qualidade do material aportado. A adição de matéria orgânica ao solo resultará em aumento ou redução do $\mathrm{pH}$ dependendo da predominância nos processos de decomposição que consomem ou liberam $\mathrm{H}^{+}$(Silva \& Mendonça, 2007). Como se trata de um solo com elevada acidez, com a adição de MOS seria esperada a liberação de bases pela decomposição e, em contrapartida, incrementos no $\mathrm{pH}$; apesar disto, o que deve ter ocorrido com a decomposição foram a liberação de prótons $\mathrm{H}^{+}$ e a complexação de base como $\mathrm{Ca}^{2+}$ (Mendonça et al., 2006).

$\mathrm{Na}$ média de $00-40 \mathrm{~cm}$ não houve diferença significativa entre os sistemas estudados nos teores de $\mathrm{H}+\mathrm{Al}$; no entanto se percebe, entre período de avaliação, efeito da sazonalização com redução no período chuvoso. Comportamento semelhante ao deste trabalho foi observado por Iwata et al. (2012) estudando SAF's na transição Cerrado/Caatinga no Piauí, que justificaram tal comportamento pela redução nos teores de MOS, tal como pôde ser observado neste trabalho.

Em geral, o comportamento da acidez trocável $\left(\mathrm{Al}^{3+}\right)$ e a saturação por $\mathrm{Al}(\mathrm{m})$, para os dois períodos de amostragem, são compatíveis com os valores de $\mathrm{pH}$ do solo. Assim, os sistemas com maior imobilização de bases em sua biomassa (MA e SAF) apresentaram os maiores valores de $\mathrm{Al}^{3+} \mathrm{e} \mathrm{H}+\mathrm{Al}$ enquanto em 
PA e RT esses parâmetros foram diminuídos, fato este atribuído aos resíduos da queima da vegetação natural. Tal comportamento é mais evidente no sistema RT na camada de $00-05 \mathrm{~cm}$ pelo uso recente do fogo (Tabela 3). Esses resultados corroboram com os de Lima et al. (2011) e Iwata et al. (2012) no que concerne à comparação entre MA e SAF porém diferem com relação a RT; esta diferença deve estar associada a sucessivos eventos de fogo relatados pelos autores o que fortalece a teoria da perda de nutrientes no sistema a cada queimada.

Ressalta-se, neste trabalho, a elevada acidez ativa, potencial, trocável e saturação por Al; nota-se, mesmo assim, expressiva produção de biomassa aérea sobretudo nos sistemas com espécies nativas ou sua predominância, tal como MA e SAF, fortalecendo a teoria de que essas espécies têm estratégia de otimização dos recursos em ambiente com elevada acidez, tal como a complexação do $\mathrm{Al}^{3+}$ pela MOS.

Em relação ao fósforo $(\mathrm{P})$, os valores decrescem no perfil do solo (Tabela 3). Esses resultados são decorrentes da baixa mobilidade no solo (Silva et al., 2011; Iwata et al., 2012) associado ao bombeamento pelas raízes das espécies cultivadas de camadas profundas do solo e posterior deposição na superfície via serrapilheira (Lima et al., 2011; Vale Júnior et al., 2011). Entre os sistemas avaliados RT apresentou os maiores valores na camada de $00-05 \mathrm{~cm}$ nos dois períodos amostrados, que é atribuído à deposição das cinzas provenientes da queima
(Menezes et al., 2008). O efeito das cinzas na disponibilidade de P não é percebido no sistema PA o que pode estar associado à sua exportação, via colheita, durante anos sucessivos de pós-queima. Em MA e SAF os menores valores em superfície são explicados pela maior biomassa, que contém proporções relativas de $\mathrm{P}$ (Lima et al., 2011).

A distribuição das bases $\mathrm{K}, \mathrm{Ca}$ e $\mathrm{Mg}$ no perfil, refletem sua associação aos teores de MOS (Tabela 4). O potássio $(\mathrm{K})$ decresce com o aumento da profundidade enquanto $\mathrm{Ca}$ e $\mathrm{Mg}$ foram maiores na camada de 00-05 $\mathrm{cm}$ nos sistemas PA e RT nos dois períodos. Variações não significativas podem ser observadas nos teores de $\mathrm{Ca}$ e $\mathrm{Mg}$ em profundidade, o que pode ser atribuído, em parte, ao efeito residual das cinzas (Menezes et al., 2008). Entre as formas de manejo do solo (nas camadas) não se observam diferenças significativas nas bases trocáveis no período seco; entretanto, no período chuvoso o $\mathrm{K}$ é maior no $\mathrm{PA}$ até $20 \mathrm{~cm}$, possivelmente associado à adição de resíduos de origem animal via deposição, tal como esterco bovino e equino, potencializados pela decomposição facilitada no período chuvoso.

Pesquisas relativamente recentes com SAF comparado a florestas nativas, têm relatado ganhos significativos de bases trocáveis na solução do solo nos sistemas manejados associados à queima da vegetação natural ou ao aporte de resíduos na superfície do solo (Menezes et al., 2008; Lima et al., 2011;

Tabela 4. Atributos químicos e físicos (AQ), potássio disponível (K), cálcio (Ca), magnésio (Mg), capacidade de troca de cátions a pH 7,0 (CTC), saturação por bases (V) e percentagem de agregados maior que $2 \mathrm{~mm}$ (Ag) nas camadas

(Z) e períodos de amostragem de um Neossolo Quartzarênico Órtico sob diferentes usos da agricultura familiar

\begin{tabular}{|c|c|c|c|c|c|c|c|c|c|c|c|c|c|c|c|c|c|}
\hline \multirow{2}{*}{$A Q$} & \multirow{2}{*}{$\begin{array}{c}Z \\
(\mathrm{~cm})\end{array}$} & \multicolumn{2}{|c|}{$M A^{1}$} & \multicolumn{2}{|c|}{ SAF } & \multicolumn{2}{|l|}{ PA } & \multicolumn{2}{|l|}{ RT } & \multicolumn{2}{|c|}{ MA } & \multicolumn{2}{|c|}{ SAF } & \multicolumn{2}{|c|}{ PA } & \multicolumn{2}{|c|}{ RT } \\
\hline & & \multicolumn{8}{|c|}{ Período seco } & \multicolumn{8}{|c|}{ Período chuvoso } \\
\hline \multirow{5}{*}{$\begin{array}{c}\mathrm{K} \\
\left(\mathrm{mg} \mathrm{dm}{ }^{-3}\right)\end{array}$} & $0-5$ & 32,36 & $\mathrm{Aa}$ & 34,71 & $\mathrm{Aa}$ & 37,21 & $\mathrm{Aa}$ & 36,93 & $\mathrm{Aa}$ & 37,21 & $\mathrm{Ba}$ & 36,71 & $\mathrm{Ba}$ & 50,57 & $\mathrm{Aa}$ & 41,14 & $\mathrm{Ba}$ \\
\hline & $5-10$ & 28,50 & $\mathrm{Ab}$ & 29,57 & $A b$ & 32,86 & $\mathrm{Aa}$ & 29,94 & $A b$ & 33,14 & $\mathrm{Bb}$ & 32,82 & $\mathrm{Bb}$ & 43,14 & $\mathrm{Aa}$ & 35,00 & $\mathrm{Bb}$ \\
\hline & $10-20$ & 27,64 & $\mathrm{Ab}$ & 26,21 & Ac & 29,93 & $\mathrm{Ac}$ & 29,14 & $A b$ & 31,86 & $\mathrm{Bb}$ & 29,79 & $\mathrm{Bc}$ & 36,64 & $\mathrm{Ab}$ & 32,29 & $\mathrm{Bb}$ \\
\hline & $20-40$ & 26,71 & $\mathrm{Ab}$ & 25,43 & Ac & 27,57 & $A b$ & 28,21 & $A b$ & 30,36 & $\mathrm{Ab}$ & 29,00 & $\mathrm{Ac}$ & 29,57 & $A b$ & 32,29 & $\mathrm{Ab}$ \\
\hline & $0-40$ & 28,80 & A & 28,98 & A & 31,89 & A & 31,06 & A & 33,14 & B & 32,09 & B & 39,98 & A & 35,18 & B \\
\hline \multirow{5}{*}{$\begin{array}{c}\mathrm{Ca} \\
\left(\mathrm{cmol}_{\mathrm{c}} \mathrm{dm}^{-3}\right)\end{array}$} & $0-5$ & 0,45 & $\mathrm{Aa}$ & 0,23 & $\mathrm{Aa}$ & 0,39 & $\mathrm{Aa}$ & 0,48 & $\mathrm{Aa}$ & 0,44 & $\mathrm{Ba}$ & 0,26 & $\mathrm{Ba}$ & 0,67 & $\mathrm{Aa}$ & 0,80 & $\mathrm{Aa}$ \\
\hline & $5-10$ & 0,24 & $\mathrm{Aa}$ & 0,25 & $\mathrm{Aa}$ & 0,29 & $A b$ & 0,29 & $A b$ & 0,30 & $\mathrm{Aa}$ & 0,35 & $\mathrm{Aa}$ & 0,34 & $A b$ & 0,29 & $A b$ \\
\hline & $10-20$ & 0,22 & $\mathrm{Aa}$ & 0,24 & $\mathrm{Aa}$ & 0,26 & $A b$ & 0,24 & $A b$ & 0,31 & $\mathrm{Aa}$ & 0,31 & $\mathrm{Aa}$ & 0,22 & $A b$ & 0,26 & $A b$ \\
\hline & $20-40$ & 0,24 & $\mathrm{Aa}$ & 0,30 & $\mathrm{Aa}$ & 0,24 & $A b$ & 0,26 & $A b$ & 0,24 & $\mathrm{Aa}$ & 0,28 & $\mathrm{Aa}$ & 0,33 & $A b$ & 0,20 & $A b$ \\
\hline & $0-40$ & 0,29 & A & 0,25 & A & 0,29 & A & 0,32 & A & 0,32 & A & 0,30 & A & 0,29 & A & 0,38 & A \\
\hline \multirow{4}{*}{$\begin{array}{c}\mathrm{Mg} \\
\left(\mathrm{cmol}_{\mathrm{G}} \mathrm{dm}^{-3}\right)\end{array}$} & $0-5$ & 0,22 & $\mathrm{Aa}$ & 0,11 & $\mathrm{Aa}$ & 0,18 & $\mathrm{Aa}$ & 0,18 & $\mathrm{Aa}$ & 0,19 & $\mathrm{Ba}$ & 0,13 & $\mathrm{Ba}$ & 0,26 & $\mathrm{Aa}$ & 0,26 & $\mathrm{Aa}$ \\
\hline & 5-10 & 0,14 & $\mathrm{Aa}$ & 0,12 & $\mathrm{Aa}$ & 0,14 & $A b$ & 0,11 & $A b$ & 0,14 & $\mathrm{Aa}$ & 0,16 & $\mathrm{Aa}$ & 0,15 & $A b$ & 0,14 & $A b$ \\
\hline & $10-20$ & 0,12 & $\mathrm{Aa}$ & 0,11 & $\mathrm{Aa}$ & 0,12 & $A b$ & 0,10 & $A b$ & 0,14 & $\mathrm{Aa}$ & 0,11 & $\mathrm{Aa}$ & 0,11 & $A b$ & 0,12 & $A b$ \\
\hline & $20-40$ & 0,12 & $\mathrm{Aa}$ & 0,14 & $\mathrm{Aa}$ & 0,11 & $A b$ & 0,13 & $A b$ & 0,11 & $\mathrm{Aa}$ & 0,12 & $\mathrm{Aa}$ & 0,14 & $A b$ & 0,10 & $A b$ \\
\hline \multirow{3}{*}{$\begin{array}{c}\text { CTC } \\
\left(\mathrm{cmol}_{\mathrm{c}} \mathrm{dm}^{-3}\right)\end{array}$} & $10-20$ & 6,70 & $A b$ & 5,46 & $\mathrm{BC}$ & 6,13 & $\mathrm{Bb}$ & 6,73 & $\mathrm{Aa}$ & 3,67 & $A C$ & 4,00 & $\mathrm{Aa}$ & 3,62 & $A b$ & 4,33 & $\mathrm{Aa}$ \\
\hline & $20-40$ & 5,22 & Ac & 4,69 & Ac & 4,71 & Ac & 5,49 & $\mathrm{Aa}$ & 2,95 & $\mathrm{Bd}$ & 2,82 & $\mathrm{Ba}$ & 3,28 & $A b$ & 3,46 & $\mathrm{Aa}$ \\
\hline & $0-40$ & 6,95 & A & 6,52 & A & 6,48 & A & 6,56 & A & 3,85 & A & 3,39 & $B$ & 3,94 & A & 4,13 & A \\
\hline \multirow{5}{*}{$\begin{array}{l}\mathrm{V} \\
(\%)\end{array}$} & $0-5$ & 8,90 & $\mathrm{Aa}$ & 5,60 & $A b$ & 9,73 & $\mathrm{Aa}$ & 12,95 & $\mathrm{Aa}$ & 15,07 & $\mathrm{Ba}$ & 12,99 & $\mathrm{Ba}$ & 27,22 & $\mathrm{Aa}$ & 28,32 & $\mathrm{Aa}$ \\
\hline & $5-10$ & 6,35 & $\mathrm{Aa}$ & 6,42 & $A b$ & 7,23 & $A b$ & 7,45 & $A b$ & 13,26 & $\mathrm{Aa}$ & 16,58 & $\mathrm{Aa}$ & 16,58 & $\mathrm{Aa}$ & 13,66 & $A b$ \\
\hline & $10-20$ & 6,36 & $\mathrm{Ba}$ & 8,07 & $A b$ & 7,89 & $A b$ & 6,45 & $\mathrm{Bb}$ & 15,41 & $\mathrm{Aa}$ & 14,73 & $\mathrm{Aa}$ & 13,66 & $A b$ & 12,56 & $A b$ \\
\hline & $20-40$ & 8,39 & $\mathrm{Aa}$ & 11,31 & $\mathrm{Aa}$ & 9,30 & $\mathrm{Aa}$ & 8,57 & $A b$ & 15,92 & $\mathrm{Aa}$ & 17,22 & $\mathrm{Aa}$ & 17,64 & $A b$ & 12,59 & $\mathrm{Bb}$ \\
\hline & $0-40$ & 7,50 & A & 7,85 & A & 8,54 & A & 8,85 & A & 14,92 & A & 15,73 & A & 18,73 & A & 16,78 & A \\
\hline \multirow{5}{*}{$\begin{array}{c}\mathrm{Ag} .>2 \mathrm{~mm} \\
(\%)\end{array}$} & $0-5$ & 77,70 & $\mathrm{Ba}$ & 88,47 & $\mathrm{Aa}$ & 71,94 & $\mathrm{Ba}$ & 68,99 & $\mathrm{Ba}$ & 69,62 & $\mathrm{Ba}$ & 80,34 & $\mathrm{Aa}$ & 55,95 & $\mathrm{Ca}$ & 44,75 & $\mathrm{Da}$ \\
\hline & 5-10 & 74,97 & $\mathrm{Aa}$ & 78,19 & $A b$ & 68,31 & $\mathrm{Aa}$ & 74,75 & $\mathrm{Aa}$ & 63,39 & $\mathrm{Aa}$ & 64,92 & $A b$ & 58,35 & $\mathrm{Aa}$ & 45,01 & $\mathrm{Ba}$ \\
\hline & $10-20$ & 77,16 & $\mathrm{Aa}$ & 72,23 & Ac & 69,07 & $\mathrm{Aa}$ & 65,56 & $\mathrm{Aa}$ & 61,77 & $\mathrm{Aa}$ & 52,82 & $\mathrm{Bc}$ & 53,46 & $\mathrm{Ba}$ & 47,03 & $\mathrm{Ba}$ \\
\hline & $20-40$ & 64,07 & $\mathrm{Bb}$ & 62,46 & $\mathrm{Bd}$ & 74,94 & $\mathrm{Aa}$ & 61,75 & $\mathrm{Ba}$ & 56,33 & $\mathrm{Aa}$ & 47,28 & Ac & 49,30 & $\mathrm{Aa}$ & 51,53 & $\mathrm{Aa}$ \\
\hline & $0-40$ & 73,48 & A & 75,34 & A & 71,07 & B & 67,76 & B & 62,78 & A & 61,34 & A & 54,27 & B & 47,08 & C \\
\hline
\end{tabular}

${ }^{1} \mathrm{MA}$ - Mata; SAF - Sistema agroflorestal; PA - Pasto; RT - Roça de toco. Médias seguidas de mesma letra maiúscula nas linhas não diferem entre sistema de uso para o mesmo período de amostragem e médias seguidas por letras minúsculas iguais nas colunas não diferem entre camadas, de acordo com os resultados do teste de Scott-Knott ( $\mathrm{p}<0,05)$. $\mathrm{N}=7$, dois anos de observação 
Silva et al., 2011; Iwata et al., 2012) embora este trabalho não tenha corroborado com tais resultados. Supõe-se, então, que tais diferenças estejam relacionadas com o tempo de manejo dos sistemas que tendem a se reequilibrar após a perturbação representada pelo desflorestamento. Neste estudo, evidências de perda de bases trocáveis pelo uso do fogo como forma de preparo do solo, só foram constatadas para $\mathrm{K}$ no período chuvoso no sistema roça de toco considerando-se que parte das bases integra a biomassa dos sistemas que, neste caso, não estão sendo contabilizadas e notadamente são mais expressivas em MA e SAF, ao contrário de RT.

Sem revolvimento, o manejo do solo proporcionou pouca alteração na MOS e, consequentemente, nos valores de CTC potencial em virtude da estreita relação entre essas variáveis (Silva et al., 2011), em especial na condição de solos muito arenosos, tal como o deste trabalho (Carneiro et al., 2009). $\mathrm{Na}$ saturação por bases (V\%), o efeito residual das cinzas só se manifestou em PA e RT na camada $00-05 \mathrm{~cm}$ no período chuvoso indicando pouca influência do manejo neste atributo do solo. Incrementos nos valores de saturação por bases no período chuvoso em relação ao período seco foram observados, fato que resulta da mineralização da MOS no período chuvoso, que reduz a CTC e, consequentemente, eleva a saturação por base tal como evidenciado por Iwata et al. (2012).
$\mathrm{O}$ uso do fogo recente em RT e o manejo em PA reduziram o tamanho dos agregados na média do perfil nos dois períodos de amostragem. Esses resultados sugerem que, em curto prazo, o tamanho dos agregados é influenciado pelo uso do fogo tal como evidenciado em RT. A longo prazo (em PA) a redução deve estar associada à carga animal (Flores et al., 2007), pois o $\mathrm{SAF}$ recebeu o mesmo preparo do solo de PA e RT e apresentou índices semelhantes à área de referência. A consequência mais relevante desses resultados é a redução na já limitada capacidade do solo em disponibilizar água às plantas e aumento da susceptibilidade à erosão (Luca et al., 2008).

Na Figura 1 pode-se observar o efeito do manejo sobre o solo ao longo do tempo de uso e espaço (camadas). Independente do período de amostragem, as maiores diferenças foram verificadas na camada de 00-05 cm (Figuras 1A e 1E), representada pela distância euclidiana necessária para formar agrupamentos entre sistemas de uso. Isto é explicado por ser a camada de maior atividade radicular das plantas potencializada pela influência da MOS na condição de solo não revolvido (Chioderoli et al., 2012). A mineralização da MOS, que é favorecida pela umidade do solo no período chuvoso (Luizão, 2007), reduz sensivelmente a capacidade de troca de cátions e libera bases para a solução do solo, o que resulta em maior variação nos atributos do solo neste período de amostragem.
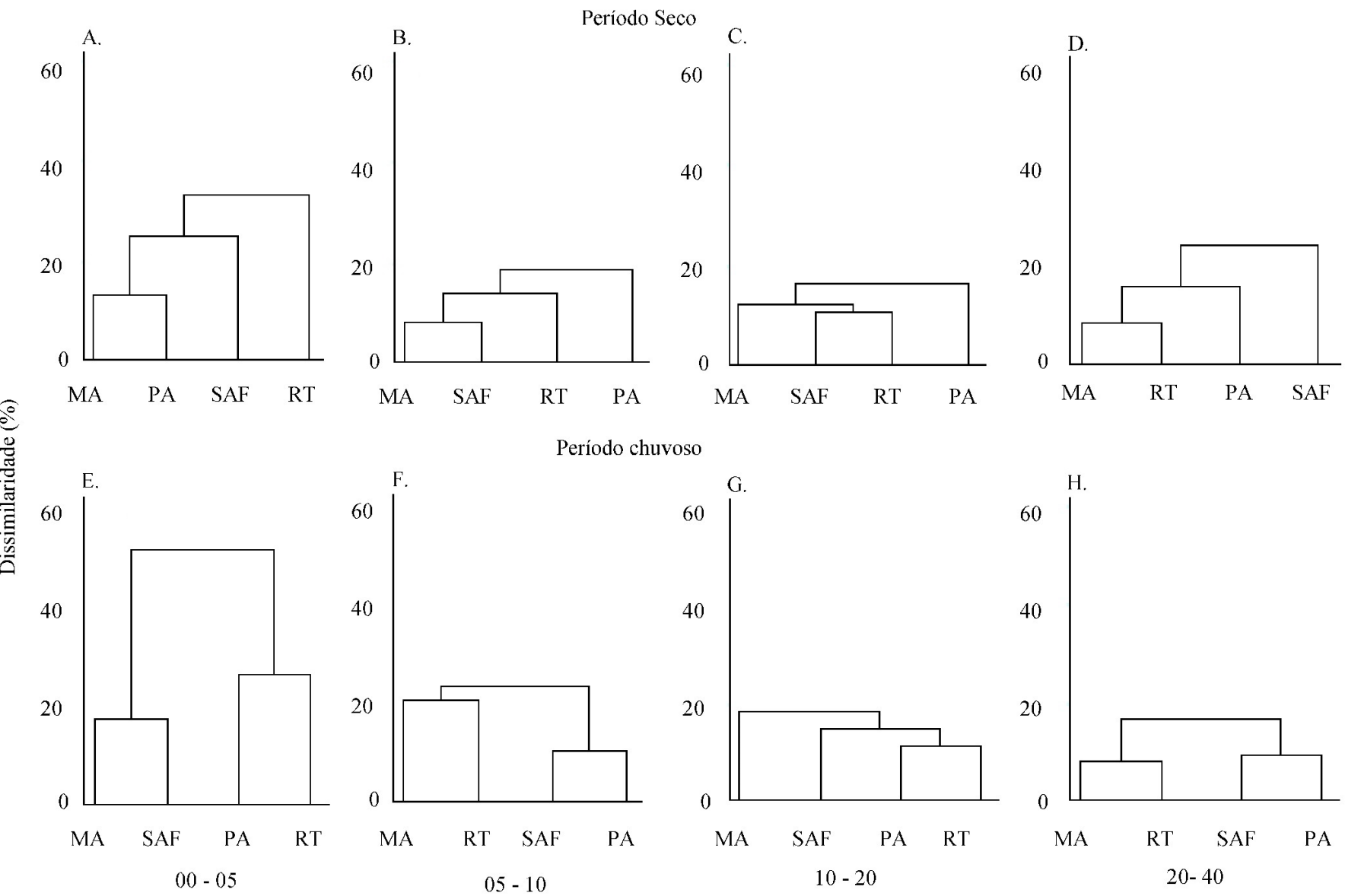

Período chuvoso
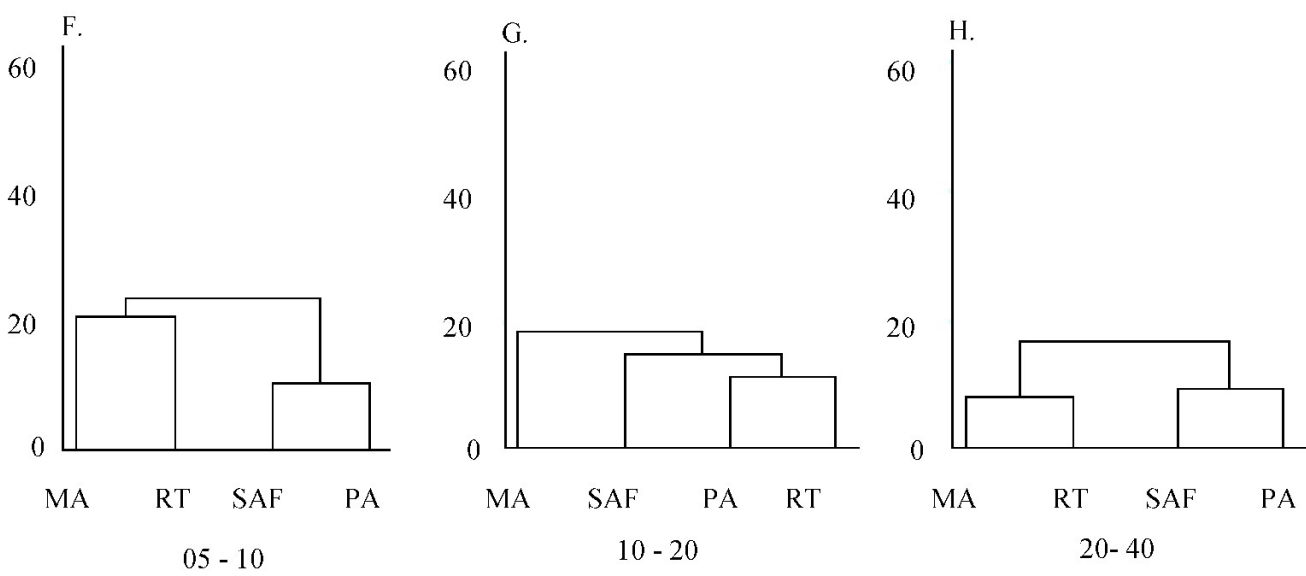

Camadas $(\mathrm{cm})$

Figura 1. Dendrograma de similaridade entre as formas de uso do solo nas camadas e períodos de amostragem de um Neossolo Quartzarênico Órtico sob diferentes usos da agricultura familiar. $N=7$, dois anos de observação. MA - Mata; SAF - Sistema agroflorestal; PA - Pasto; RT - Roça de toco 
A semelhança do sistema RT com a área de referência na camada de 20-40 cm (Figuras 1D e 1H) indica que o uso do fogo não alterou significativamente os atributos do solo em profundidade. No entanto, na superfície $(00-05 \mathrm{~cm})$ proporcionou melhorias decorrentes das cinzas provenientes da combustão da biomassa (Iwata et al., 2012), afastando-se da área de referência. Esta fertilização superficial do solo por meio da queima da vegetação natural tem sido o principal atrativo da agricultura itinerante do trópico úmido (Moura, 2004).

Em geral, a qualidade dos indicadores químicos do solo se relacionou com o acúmulo de biomassa aérea dos sistemas de uso. Assim, a área de referência (MA) apresentou os piores indicadores químicos do solo, tendo em vista que grande parte dos nutrientes se encontrava imobilizada na sua biomassa (Luizão, 2007). Isto sugere ser uma forma de proteção eficiente dos nutrientes dos efeitos do clima na lixiviação, sobretudo em solos arenosos, como os da Amazônia (Ferreira et al., 2006).

\section{Conclusões}

1. Vinte e dois anos após a conversão da floresta em agroecossistema, por meio do corte e queima da vegetação natural, o sistema agroflorestal ainda apresenta indicadores de qualidade do solo semelhantes à área de mata evidenciando a longevidade desta forma de uso do solo.

2. O sistema de uso com pastagem, por apresentar cobertura vegetal com menos biomassa, mostra-se com melhores indicadores químicos que a mata e sistema agroflorestal, o que decorre do efeito residual das cinzas proveniente da queima da floresta.

3. No sistema roça de toco, o efeito do fogo só é evidenciado nos primeiros $5 \mathrm{~cm}$ de profundidade do solo, o que pode ser atribuído ao curto período de uso.

4. Este estudo indica que a agricultura familiar conduzida de forma primitiva não causa impactos negativos nos atributos químicos do solo. A proteção da matéria orgânica do solo associada ao não revolvimento do solo e às baixas saídas de nutrientes dos sistemas, representadas pelas colheitas, são, possivelmente, as principais razões para tais resultados.

\section{Agradecimentos}

Os autores agradecem a Damião Paulino de Brito e à $\mathrm{Sr}^{\mathrm{a}}$ Francisca, proprietários do lote objeto da pesquisa, pelo apoio físico; à Coordenação de Aperfeiçoamento de Pessoal de Nível Superior (CAPES), pelo apoio financeiro com bolsa de estudo PIQDTEC ao primeiro autor e ao Conselho Nacional de Desenvolvimento Científico e Tecnológico ( $\mathrm{CNPq}$ ) pela Bolsa de estudo ao segundo autor.

\section{Literatura Citada}

Burak, D. L.; Passos, R. R. Variabilidade espacial das frações texturais e da porosidade do solo em relevo ondulado sob cultivo de café conilon. Enciclopédia Biosfera, v.7, p.1-7, 2011.
Caldeira, M. V. W.; Marques, R.; Soares, R. V.; Balbinot, R. Quantificação de serrapilheira e de nutrientes em floresta ombrófila mista Montana-Paraná. Revista Acadêmica, v.5, p.101-116, 2007.

Carneiro, M. A. C.; Souza, E. D.; Reis, E. F.; Pereira, H. S.; Azevedo, W. R. Atributos físicos, químicos e biológicos de solos de Cerrado sob diferentes sistemas de uso e manejo. Revista Brasileira de Ciência do Solo, v.33, p.147-157, 2009.

Cey, E. E.; Rudolph, D. L.; Passmore, J. Influence of macroporosity on preferencial solute and colloid transport in unsaturated field soils. Journal of Contaminant Hydrology, v.107, p.45-57, 2009.

Chioderoli, C. A.; Mello, L. M. M.; Grigolli, P. J.; Furlani, C. E. A.; Silva, J. O. R.; Cesarin, A. L. Atributos físicos do solo e produtividade de soja em sistema de consórcio milho e brachiaria. Revista Brasileira de Engenharia Agrícola e Ambiental, v.16, p.37-43, 2012.

Dubois, J. C. L.; Viana, V. M.; Anderson, A. B. Sistemas e práticas agroflorestais para Amazônia. Manual agroflorestal para a Amazônia. Rio de Janeiro: REBRAF, 1996. v.1, p.2-27.

EMBRAPA - Empresa Brasileira de Pesquisa Agropecuária. Centro Nacional de Pesquisa de Solos. Manual de métodos de análises de solos. 2.ed. Rio de Janeiro: Embrapa Solos, 1997. 212p.

EMBRAPA - Empresa Brasileira de Pesquisa Agropecuária. Centro Nacional de Pesquisa de Solos. Sistema brasileiro de classificação de solos. 2.ed. Rio de Janeiro: Embrapa Solos, 2006. 306p.

Ferreira, S. J. F.; Luizão, F. J.; Miranda, S. A. F.; Silva, M. S. R.; Vital, A. R. T. Nutrientes na solução do solo em floresta de terra firme na Amazônia central submetida à extração seletiva de madeira. Acta Amazonica, v.36, p.59-68, 2006.

Flores, J. P. C.; Anchinoli, I.; Cassol, L. C.; Carvalho, P. C. F.; Leite, J. G. B.; Fraga, T. I. Atributos físicos do solo e rendimento de soja em sistema plantio direto em integração lavoura-pecuária com diferentes pressões de plantio. Revista Brasileira de Ciência do Solo, v.31, p.771-780, 2007.

Gehring, C. O ambiente do trópico úmido e o manejo sustentável dos agroecossistemas. In: Moura, E. G.; Aguiar, A. C. F. (org.). O desenvolvimento rural como forma de ampliação dos direitos no campo: Princípios e tecnologias. 1. ed. Série Agroecologia, São Luís: UEMA, 2006. v.2. cap. 6, p.101-129.

Hammer, O.; Harper, D. A. T.; Ryan, P. D. PAST: Paleontological statistics software package for education and data analysis. Paleontologia Electronica, v.4, p.1-9, 2001.

Iwata, B. F.; Leite, L. F. C.; Araújo, A. S. F.; Nunes, L. A. P. L.; Gehring, C.; Campos, L. P. Sistemas agroflorestais e seus efeitos sobre os atributos químicos em Argissolo Vermelho-Amarelo do Cerrado piauiense. Revista Brasileira de Engenharia Agrícola e Ambiental, v. 16, p.730-738, 2012.

Lima, S. S.; Leite, L. F. C.; Oliveira, F. C.; Costa, D. B. Atributos químicos e estoque de carbono e nitrogênio em Argissolo Vermelho-Amarelo sob sistemas agroflorestais e agricultura de corte e queima no norte do Piauí. Revista Árvore, v.35, p.51-60, 2011. 
Luca, E. F.; Feller, C.; Cerri, C. C.; Barthes, B.; Chaplot, V.; Campos, D. C. C.; Manechini, C. Avaliação de atributos físicos e estoque de carbono e nitrogênio em solos com queima e sem queima de canavial. Revista Brasileira de Ciência do Solo, v.32, p.789-800, 2008.

Luizão, F. J. Ciclos de nutrientes na Amazônia: Respostas às mudanças ambientais e climáticas. Ciência e Cultura, v.57, p.31-36, 2007.

Luizão, F. J.; Tapia-Coral, S.; Gallardo-Ordinola, J.; Silva, G. C.; Luizão, R. C. C.; Trufillo-Cabrera, L.; Wandelli, E.; Fernandes, E. C. M. Ciclos biogeoquímicos em Agrofloresta na Amazônia. In: Gama-Rodrigues, A. C.; Barros, N. F.; Gama-Rodrigues, E. F.; Freitas, M. S. M. (org.). Sistemas agroflorestais: bases para o desenvolvimento sustentável. Campos dos Goytacazes: Universidade Estadual do Norte Fluminense Darcy Ribeiro, 2006. p.87-100.

Maia, S. M. F.; Xavier, F. A. S.; Oliveira, T. S.; Mendonça, E. S.; Araújo Filho, J. A. Impacto de sistemas agroflorestais e convencional sobre a qualidade do solo no semi-árido cearense. Revista Árvore, v.33, p.837-848, 2006.

Matoso, S. C. G.; Silva, A. N.; Fiorelli-Pereira, E. C.; Colleta, Q. P.; Maia, E. Frações de carbono e nitrogênio de um Latossolo Vermelho-Amarelo distrófico sob diferentes usos da Amazônia brasileira. Acta Amazônica, v.42, p.231-240, 2012.

Mendonça, E. S.; Rowell, D. L.; Martins, A. G.; Silva, A. P. Effect of $\mathrm{pH}$ on the development of acidic sites in clayey and Sandy loam Oxisol from the Cerrado Region, Brazil. Geoderma, v.132, p.131-142, 2006.
Menezes, J. M. T.; Leeuwen, J. V.; Valeri, S. V.; Cruz, M. C. P.; Leandro, R. C. Comparação entre solos sob uso agroflorestal e em florestas remanescentes adjacentes, no norte de Rondônia. Revista Brasileira de Ciência do Solo, v.32, p.893-898, 2008.

Moura, E. G. Agroambientes de transição avaliados numa perspectiva da agricultura familiar. In: Moura, E. G. (org.). Agroambientes de transição entre o trópico úmido e o semiárido do Brasil. São Luís: UEMA, 2004. cap. 1, p.15-51.

Pedron, F. A.; Fink, J. R.; Rodrigues, M. F.; Azevedo, A. C. de. Condutividade e retenção de água em Neossolos e saprolitos derivados de arenito. Revista Brasileira de Ciência do Solo, v.35, p.1253-1262, 2011.

Sanches, L.; Valentini, C. M. A.; Biudes, M. S.; Nogueira, J. S. Dinâmica sazonal da produção e decomposição de serrapilheira em floresta tropical de transição. Revista Brasileira de Engenharia Agrícola e Ambiental, v.13, p.183189, 2009.

Silva, D. C.; Silva, M. L. N.; Curi, N.; Oliveira, A. H.; Souza, F. S.; Martins, S. G.; Macedo, R. L. G. Atributos do solo em sistemas agroflorestais, cultivo convencional e floresta nativa. Revista de Estudos Ambientais, v.13, p.77-86, 2011.

Silva, I. R.; Mendonça, E. S. Matéria orgânica do solo. In: Novais, R. F.; Alvarez, V. V. H.; Barros, N. F.; Fontes, R. L. F.; Cantarutti, R. B.; Neves, J. C. L. (org.). Fertilidade do solo. 1017p. Viçosa: Sociedade Brasileira de Ciência do Solo, 2007. cap. 6, p.275-374.

Vale Júnior, J. F.; Souza, M. I. L.; Nascimento, P. R. R.; Cruz, D. L. S. Solos da Amazônia: Etnopedologia e desenvolvimento sustentável. Revista Agro@mbiente, v.5, p.158-165, 2011. 